\title{
Parents' Knowledge of Neonatal Danger Signs and Associated Factors at Health Centers in Kigali, Rwanda
}

\author{
Patricie Mujawimana ${ }^{1 *}$, Fauste Uwingabire $^{1}$, Felicite Kankindi $^{1}$, Ruth Dusabe ${ }^{1}$, Pamela \\ Meharry $^{2}$ \\ ${ }^{1}$ School of Nursing and Midwifery, University of Rwanda, College of Medicine and Health \\ Sciences, Kigali, Rwanda \\ ${ }^{2}$ Department of Women's, Children's and Family Health Services, University of Mlinois, Chicago, \\ USA
}

*Corresponding author: Patricie Mujawimana. School of Nursing and Midwifery, College of Medicine and Health Sciences, University of Rwanda, Rwamagana Campus, Eastern Province, Rwanda. Email: mujapaty@gmail.com.

\begin{abstract}
Background

Globally, nearly half of all under-five deaths occur during the neonatal period. About two million dies within the first week, of which $75 \%$ come from low-resource countries, such as Rwanda. Many neonatal deaths are preventable or avoidable if parents are knowledgeable of Neonatal Danger Signs (NDS), and do not delay seeking care at a health facility.

Objective

To assess the parents' knowledge of NDS and associated factors within the neonatal period at four health centers in Kigali.
\end{abstract}

\section{Method}

This study was a descriptive cross-sectional design. A proportionate stratified probability sampling strategy was used to select 209 parents who attended selected health centers in Kigali. Data analysis used descriptive and inferential statistics.

\section{Results}

The findings showed that $67 \%$ of participants had some information on NDS. Logistic regression showed that educational level, parity, number of antenatal visits, and information from healthcare providers was significantly associated with parents' knowledge of NDS.

\section{Conclusion}

Our findings indicate the need to enhance education of parents' knowledge of NDS in the study population. Educational efforts also should target NDS in health centers where most Rwandan women attend antenatal care.

Rwanda J Med Health Sci 2020;3(2):128-138

\section{Keywords: Neonatal, danger signs, parents, knowledge, associated factors, Rwanda}

\section{BACKGROUND}

Approximately $47 \%$ of child under- 5 deaths occur during the neonatal period, a proportion that has been trending upwards since 1990.[1] The majority of neonatal deaths $(75 \%)$ occur in the first week after birth, with one million deaths on the first day, and a total of 2.5 million over the first 28 days after birth.[1] In 2018, the Sub-Sahara Africa (SSA) region had the highest rate of neonatal deaths at 28 per 1,000 live births.[1] Many neonates in SSA die from preventable or treatable causes;[2] one major modifiable factor is the late recognition of danger signs of illness by parents and caregivers, and delay in decision-making to seek health care at the onset of illness signs.[3]

Although many African countries made substantial progress in decreasing child deaths during the Millennium Development Goals (MDGs) era, including Rwanda, neonatal deaths did not decline 
at the rate of other under-5 groups.[4] Globally, birth asphyxia, preterm birth, birth defects, and infections caused the most neonatal deaths in 2017.[1] A recent retrospective study in Rwanda, by Uwingabire and Gowan,[5] found a 40\% birth asphyxia rate among neonates admitted to the Neonatal Intensive Care Unit (NICU). A recent matched case-control study in Rwanda also found pneumonia and meningitis are common causes of neonatal mortality, with nearly a third dying at home.[6]

A systematic review involving 34 studies in 17 SSA countries, revealed that half of the children under5 died at home.[7] Nearly all (95\%) had signs and symptoms of illness, but care-seeking behaviour differed with the child's age: " $40.1 \%$ of neonates died without receiving any care, compared to $6.4 \%$ of older children".[7] The review authors cited "poor illness recognition" was associated with high neonatal mortality.[7] The World Health Organization (WHO) identified danger signs that families should be aware of in order to identify neonatal illness and seek timely care.[1] Neonatal danger signs (NDS) include: feeding poorly or not at all, reduced or no movement, difficulty breathing, fever or feels cold, and convulsions.[1] The WHO also provides detailed essential newborn care guidelines to alert healthcare providers (HCP) of NDS, such as temperature $\geq 38.0^{\circ} \mathrm{C}$ or $<35.5^{\circ} \mathrm{C}$. [8] The neonatal period represents a critical survival phase when parents and caregivers should be equipped with the knowledge to recognize NDS and seek timely care at a health facility to achieve the best possible neonatal health outcomes.

Several recent studies conducted in SSA indicated that the majority of new mothers had low awareness of NDS, which delayed seeking care.[9-11] The authors recommended NDS education during antepartum and postpartum visits; postnatal care, such as vaccination clinics; and the use of a Maternal Child Health Booklet that parents could read or look at pictures of NDS at home.[9-11] There does not appear to be a study conducted in Rwanda on parents "knowledge of NDS.

The Sustainable Development Goal 3 (SDG3) has a target of $12 \leq$ neonatal deaths per 1,000 live births by 2030.[2] Rwanda reported a neonatal mortality rate of $29 / 1000$ live births in 2015, which contributed to $40 \%$ of under-5 mortality.[4] The investigator of this study selected this topic to make a local challenge more visible, and subsequently help Rwanda achieve the SDG3.[12] Understanding parents' knowledge of NDS and their care-seeking patterns enable targeted interventions to reduce mortality.[7] This study aimed to assess mothers' knowledge of NDS at four health centers in Rwanda.

\section{METHODS}

\section{Design}

A descriptive cross-sectional study design was used to assess the parents' knowledge of NDS and associated factors. We gathered data at four health centers located in the Muhima District Hospital catchment area, in Kigali, Rwanda. Two health centers were located in the urban area (Cornum and Kabusunzu), and the two were located in the suburban area (Rugarama and Butamwa). The study was conducted from February 22 to April 19, 2019.

\section{Participants' recruitment}

The sampling strategy was a proportionate stratified sampling of parents $\geq 18$ years with a living neonate in the first 28 days post birth. A proportional number of participants from each health center was selected depending on the size of the population, whereby 67 were from Cornum (Kimisagara), 48 from Kabusunzu, 52 from Rugarama, and 42 from Butamwa. The total sample size was 209 parents, using Solvin's formula, a confidence interval of $95 \%$, and a margin error of $5 \%$. The study population involved all parents with neonates who attended the vaccination services.

\section{Measures}

A self-administered questionnaire with closedended questions was used to collect data. The questionnaire was modified from a study done by Sandberg and colleagues in Southwestern Rural Uganda.[11] Permission to adapt and use the questionnaire was provided by the corresponding lead author, via email on October 23, 2018. The questionnaire was adapted to the local language, Kinyarwanda, in order to facilitate the participants' understanding. The questionnaire had four sections and included; socio-demographic characteristics, obstetrical factors, parents' knowledge of NDS, and source of information.

Section one assessed the sociodemographics characteristics with six items; age, education level, religion, occupation, and place of residence. These variables were measured using frequencies (Table $1)$. 
Section two assessed the obstetrical factors with four items; parity, number of antenatal visits, place of delivery, and days since delivery. These variables were measured using frequencies (Table 2).

Section three included participants who heard information about NDS (Yes or No) and the source of information with five items: HCP, community health workers (CHW), radio, television (TV), and neighbors. These variables were measured using frequencies for awareness of NDS and the source of information (Figure 1).

Section four consisted of parental knowledge of listed NDS. It included 16 items: not feeding well, convulsions, severe chest in-drawing, temperature $>37.5^{\circ} \mathrm{C}$, temperature $<35.5^{\circ} \mathrm{C}$, no spontaneous movement, fast breathing ( $\geq 60$ breaths $/ \mathrm{min}$ ), grunting, slow breathing or gasping $1<30$ breaths/min); heart rate constantly > 180/min, floppy or stiff, umbilicus draining pus, redness and swelling extending to skin; bleeding from umbilicus or cut; $\geq 10$ skin pustules or bullae, or swelling, redness, hardness of skin; pallor and diffuse cyanosis. These variables were measured through frequencies (Table 3). The data collector only ticked what the participant mentioned only, the range from 0 up to $\geq 4$ indicate the number of NDS recognized by the participants.

The tool was pretested on 21 participants from the four health centers completed the questionnaire for feasibility purposes. The participants involved in the pilot test were not included in the final results. A Cronbach's Alpha was 0.78 indicated the internal consistency of the instrument.

\section{Data collection}

Individual participants were approached at the vaccination services at the four health centers. The study was explained to the participants, and they were invited to participate, knowing that their responses would be confidential, anonymous, and they could withdraw at any time. Permission was obtained through written consent before data collection. A structured questionnaire was administered to individual parents and was completed in about 15 minutes, or longer for those needing more assistance. The investigator helped those who did not know how to read or write by reading the question and documenting the participant's selected response. The investigator was responsible for distributing and collecting the completed questionnaires.

\section{Data analysis}

The data were coded, entered, and analyzed using SPSS software version 24.0. Socio-demographic and obstetrical factors data were summarized using descriptive statistics, namely frequencies and percentages. Variables which showed significant association in Bivariate analysis were recruited in Logistic regression to analyze predictors of mothers' knowledge of NDS. Bivariate analysis was carried out to assess the relationship between independent variables and knowledge of NDS. Logistic regression test was used to predict parents' knowledge of NDS and associated factors. Statistical significance was defined as a $p$-value of $\leq 0.05$.

\section{Ethical considerations}

Permission to conduct this study was obtained from the Institutional Review Board of the University of Rwanda, College of Medicine and Health Sciences. In addition, approval was obtained from the internal ethical committee of the Muhima District Hospital, which oversees the four health centers. All participants signed the consent form prior to data collection.

\section{RESULTS}

Characteristics of participants sociodemographic A total of 209 new mothers who attended the vaccination clinics at one of the four health centers completed the study. The data collection occurred from February 22 to April 19, 2019. 
Table 1. Sociodemographic characteristics of participants $(\mathbf{n}=209)$

\begin{tabular}{|c|c|}
\hline Variables & n (\%) \\
\hline \multicolumn{2}{|l|}{ Age (Years) } \\
\hline $18-20$ & $20(9.6)$ \\
\hline $21-26$ & 55 (26.3) \\
\hline $27-32$ & $56(26.8)$ \\
\hline $33-38$ & $49(23.4)$ \\
\hline$\geq 39$ & 29 (13.9) \\
\hline \multicolumn{2}{|l|}{ Marital status } \\
\hline Married & $100(47.8)$ \\
\hline Single & $24(11.5)$ \\
\hline Divorced & $3(1.4)$ \\
\hline Separated & $9(4.3)$ \\
\hline Widower & $2(1.0)$ \\
\hline Cohabitating & $71(34.0)$ \\
\hline \multicolumn{2}{|l|}{ Religion } \\
\hline Catholic & $62(29.7)$ \\
\hline Protestant & 95 (45.5) \\
\hline Muslims & $23(11.0)$ \\
\hline Adventist & 29 (13.9) \\
\hline \multicolumn{2}{|l|}{ Education level } \\
\hline Unable to read and write & $14(6.7)$ \\
\hline Able to read and write & $17(8.1)$ \\
\hline Primary school & 96 (45.9) \\
\hline Ordinal level & $23(11.0)$ \\
\hline Secondary school & $49(23.4)$ \\
\hline University & $10(4.8)$ \\
\hline \multicolumn{2}{|l|}{ Occupation } \\
\hline Housewife and mother & $105(50.2)$ \\
\hline Daily laborer & $44(21.1)$ \\
\hline Merchant & $58(27.8)$ \\
\hline Civil servant & $1(0.5)$ \\
\hline Owns private business & $1(0.5)$ \\
\hline \multicolumn{2}{|l|}{ Place of Residence } \\
\hline Urban & $140(67.0)$ \\
\hline Sub-urban & $60(28.7)$ \\
\hline Rural & $9(4.3)$ \\
\hline
\end{tabular}

The socio-demographic characteristics of participants were presented in table 1 . The majority of participants were aged between 27-32 years (26.8\%) followed by those who were between 21-26 years (26.3\%). The majority were married (47.8\%). Most participants (45.5\%) were protestant affiliation. The majority completed primary school (45.9\%). Participants were asked their occupation, and over half $(50.2 \%)$ reported that they were housewives, those who live in urban area (67\%). 


\section{Obstetrical factors of participants}

Table 2. Obstetrical factors of participants $(\mathbf{n}=209)$

\begin{tabular}{lc}
\hline Variables & n (\%) \\
\hline Parity & $64(30.6)$ \\
1 & $54(25.8)$ \\
2 & $48(23.0)$ \\
3 & $25(12.0)$ \\
4 & $14(6.7)$ \\
5 & $4(1.9)$ \\
$\geq 6$ & $1(0.5)$ \\
ANC visits & $24(11.5)$ \\
0 & $33(15.8)$ \\
1 & $98(46.9)$ \\
2 & $53(25.4)$ \\
3 & \\
4 & $181(86.6)$ \\
Days since delivery & $28(13.4)$ \\
$1-14$ & \\
$15-28$ & $3(1.4)$ \\
Place of delivery & $103(49.3)$ \\
Home & $96(45.9)$ \\
Heath center & $7(3.3)$ \\
Public hospital & \\
Private hospital & \\
\hline
\end{tabular}

The obstetrical factors of participants are presented in Table 2 . The majority $(30.6 \%)$ reported this was their first pregnancy, and attending their third ANC visit (46.9\%). The majority (49.3\%) reported that they delivered at the health center and that it was 1-14 days since the date of delivery (86.6\%). 


\section{Source of NDS information}

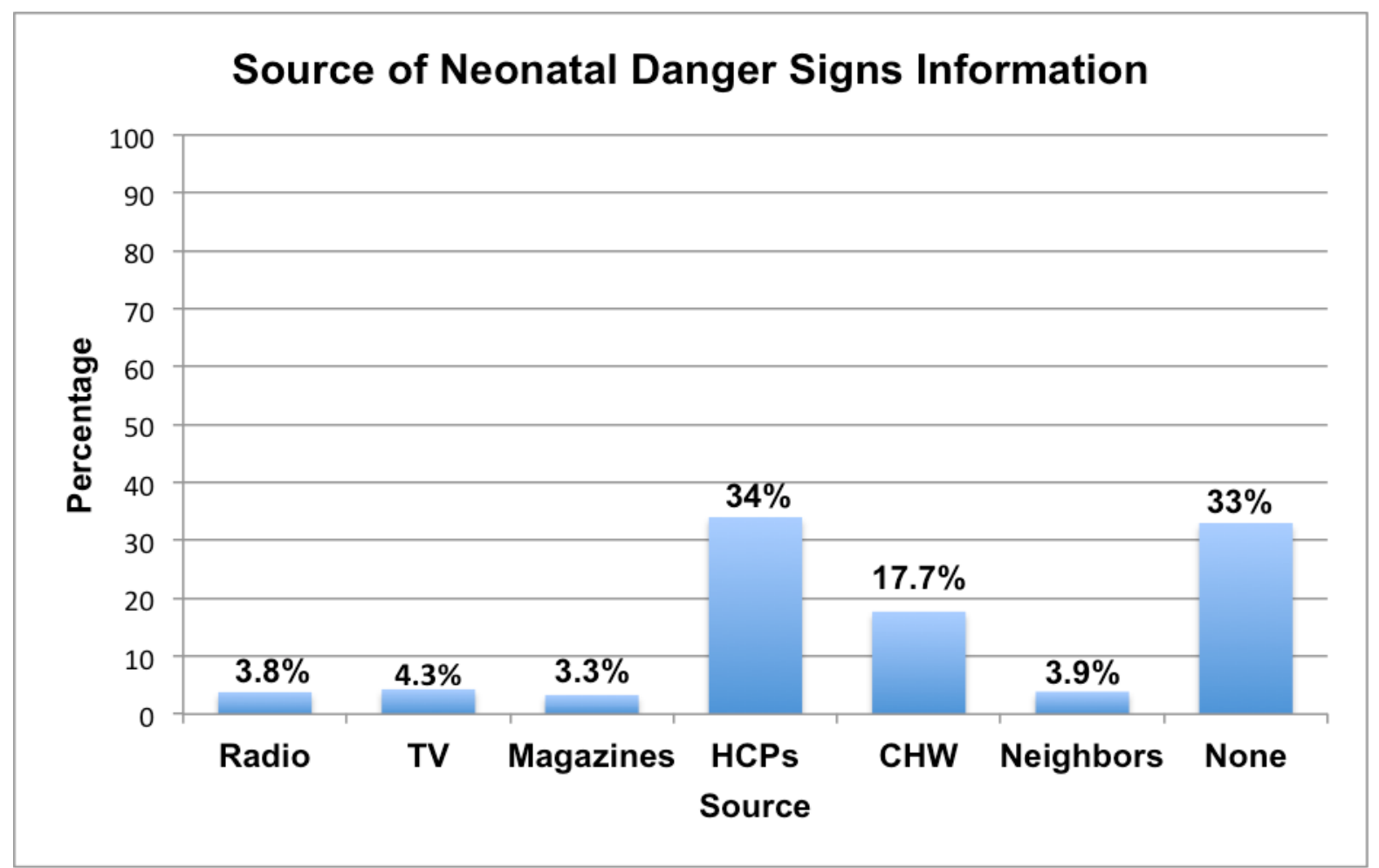

Figure 1. Source of NDS information

The source of information on knowledge about NDS was displayed in figure 2 . The majority (34\%) received information from the HCP; however, a good proportion of participants $(17.7 \%)$ received

\section{Parental knowledge of neonatal danger signs}

Results of parental knowledge of NDS were presented in table 3. Participants were asked if they were aware of NDS, and results showed that (67\%) responded, "Yes," they were aware of NDS. The top information from $\mathrm{CHW}$, though a limited number received information from TV (4.3\%), neighbors $(3.9 \%)$, radio $(3.8 \%)$, and magazines $(3.3 \%)$.

three NDS danger signs that parents were aware of included; not feeding (44.0\%), high body temperature $(24.9 \%)$, and convulsions $(22.0 \%)$. 
Table 3. Parental knowledge of neonatal danger signs $(n=140)$

\begin{tabular}{llc}
\hline \multirow{2}{*}{ Neonatal Danger Signs } & \multicolumn{2}{c}{ Parental knowledge of NDS } \\
Yes n (\%) & No n (\%) \\
\hline Not feeding well & $99(44.0)$ & $117(56.0)$ \\
Convulsions & $46(22.0)$ & $163(78.0)$ \\
Severe chest in drawings & $5(2.4)$ & $204(97.6)$ \\
Temperature $>37.5^{\circ} \mathrm{C}$ & $52(24.9)$ & $157(75.1)$ \\
Temperature $<35.5^{\circ} \mathrm{C}$, or not rising after rewarming & $10(4.8)$ & $199(95.2)$ \\
No spontaneous movement, floppy or stiff & $2(1.0)$ & $207(99.0)$ \\
Fast breathing $(>60$ bpm) & $14(6.7)$ & $195(93.3)$ \\
Slow breathing or gasping $(<30$ bpm) & $8(3.8)$ & $201(96.2)$ \\
Grunting & $6(2.9)$ & $203(97.1)$ \\
Diffuse cyanosis & $13(6.2)$ & $196(93.8)$ \\
Heart rate constantly $>180 /$ min & $2(1.0)$ & $207(99.0)$ \\
Umbilicus drains pus, redness and swelling extends to skin & $9(4.3)$ & $200(95.7)$ \\
$>10$ skin pustules or bullae, or swelling, redness, hard skin & $2(1.0)$ & $207(99.0)$ \\
Bleeding from umbilicus or cut & $11(5.3)$ & $198(94.7)$ \\
Pallor & $18(8.6)$ & $191(91.4)$ \\
\end{tabular}

\section{Association between independent variables and knowledge of NDS}

Table 4. Bivariate analysis between independent variables and knowledge of NDS

\begin{tabular}{|c|c|c|c|c|c|c|}
\hline \multirow[t]{2}{*}{ Variables } & \multicolumn{6}{|c|}{$\begin{array}{l}\text { Awareness of Neonatal Danger Signs (from } 0 \text { - } \geq 4 \text { : Number of NDS recognized by the } \\
\text { participants) }\end{array}$} \\
\hline & $\mathbf{0}$ & 1 & 2 & 3 & $\geq 4$ & $\mathbf{P}$ \\
\hline \multicolumn{6}{|l|}{ Education level } & 0.05 \\
\hline Cannot read \& write & $3(21.4 \%)$ & $4(28.6 \%)$ & $5(35.7 \%)$ & $2(14.3 \%)$ & $0(0.0 \%)$ & \\
\hline Can read and write & $11(64.7 \%)$ & $3(17.6 \%)$ & $1(5.9 \%)$ & $2(11.8 \%)$ & $0(0.0 \%)$ & \\
\hline Primary school & $30(31.3 \%)$ & $2(22.9 \%)$ & $31(32.3 \%)$ & $8(8.3 \%)$ & $5(5.3 \%)$ & \\
\hline Ordinal level & $9(30.4 \%)$ & $3(13.0 \%)$ & 7 (30.4\%) & $6(21.1 \%)$ & $0(0.0 \%)$ & \\
\hline Secondary school & $12(24.5 \%)$ & $7(14.3 \%)$ & $13(26.5 \%)$ & $12(24.5 \%)$ & $5(10.2 \%)$ & \\
\hline University & $6(60.0 \%)$ & $0(0.0 \%)$ & $2(20.0 \%)$ & $2(20.0 \%)$ & $0(0.0 \%)$ & \\
\hline \multicolumn{6}{|l|}{ ANC visits } & 0.02 \\
\hline 0 & $1(100.0 \%)$ & $0(0.0 \%)$ & $0(0,0 \%)$ & $0(0.0 \%)$ & $0(0.0 \%)$ & \\
\hline 1 & $12(50 \%)$ & $2(8.35)$ & $5(28.8 \%)$ & $4(16.7 \%)$ & $1(4.2 \%)$ & \\
\hline 2 & $10(62.6 \%)$ & $3(9.1 \%)$ & $12(36.4 \%)$ & $6(18.2 \%)$ & $2(6.1 \%)$ & \\
\hline 3 & $14(14.3 \%)$ & $33(33.7 \%)$ & 27 (27.6\%) & $21(21.4 \%)$ & $3(3.1 \%)$ & \\
\hline$\geq 4$ & $4(7.5 \%)$ & $8(15.1 \%)$ & $9(17.0 \%)$ & $13(24.5 \%)$ & $19(35.8 \%)$ & \\
\hline \multicolumn{6}{|l|}{ Place of delivery } & 0.03 \\
\hline Home & $1(33.3 \%)$ & $2(66.7 \%)$ & $0(0.0 \%)$ & $0(0.0 \%)$ & $0(0.0 \%)$ & \\
\hline Health center & $14(13.6 \%)$ & $30(29.1 \%)$ & $31(30.1 \%)$ & $24(23.3 \%)$ & $4(3.9 \%)$ & \\
\hline Public hospital & $17(17.7 \%)$ & $35(36.5 \%)$ & $27(28.1 \%)$ & $13(13.5 \%)$ & $4(4.2 \%)$ & \\
\hline \multicolumn{7}{|l|}{ Private hospital } \\
\hline Parity & & & & & & 0.02 \\
\hline 1 & 26 (40.6\%) & $10(15.6 \%)$ & $16(25.0 \%)$ & 10 (15.6\%) & $2(3.1 \%)$ & \\
\hline $2-4$ & $18(14.3 \%)$ & $39(31.0 \%)$ & $36(28.6 \%)$ & $27(21.4 \%)$ & $6(4.8 \%)$ & \\
\hline$\geq 5$ & $2(10.5 \%)$ & $2(10.5 \%)$ & $4(21.1 \%)$ & $4(21.1 \%)$ & 7 (36.8\%) & \\
\hline \multicolumn{7}{|l|}{ Information source } \\
\hline Radio & 2 (28.6\%) & 3 (42.9\%) & $2(28.6 \%)$ & $0(0.0 \%)$ & $0(0.0 \%)$ & 0.45 \\
\hline TV & $3(33.4 \%)$ & $4(44.4 \%)$ & $1(11.1 \%)$ & $11(11.1 \%)$ & $0(0.0 \%)$ & 0.21 \\
\hline $\mathrm{HCP}$ & $1(1.4 \%)$ & $13(18.3 \%)$ & $34(47.9 \%)$ & $16(22.5 \%)$ & 7 (9.9\%) & 0.02 \\
\hline CHW & $3(8.3 \%)$ & $9(25.0 \%)$ & $13(36.1 \%)$ & $9(25 \%)$ & $2(5.6 \%)$ & 0.01 \\
\hline
\end{tabular}


Bivariate analysis was conducted to analyze the association between the independent variables and knowledge of NDS (table 4). Education level ( $p=0.047)$, the number of ANC visits $(p=0.021)$, place of delivery $(\mathrm{p}=0.027)$, and parity $(\mathrm{p}=0.016)$ showed a statistically significant association with knowledge of NDS. Two sources of information HCP $(p=0.015)$, and CHW $(p=0.013)$ also showed association with knowledge of NDS

Table 5. Logistic regression of parents' knowledge of NDS with Socio demographic characteristics

\begin{tabular}{lccc}
\hline Variables & $\boldsymbol{\beta}$ & $\mathbf{9 5 \%} \mathbf{C I}$ & $\mathbf{p}-$ value \\
& & & \\
\hline Education level & & & \\
Unable to read and write (ref) & 1.345 & $1.121-1.786$ & 0.25 \\
Able to read and write & 1.745 & $1.622-1.981$ & 0.01 \\
Primary school & 2.245 & $1.924-2.882$ & \\
Secondary and above & & & 0.04 \\
Parity & 1.978 & $1.675-2.322$ & 0.03 \\
1 (ref) & 2.118 & $1.975-2.722$ & \\
$2-4$ & & & 0.23 \\
$\geq 5$ & 1.432 & $1.242-1.767$ & 0.02 \\
ANC visits & 1.987 & $1.764-2.769$ & 0.01 \\
1 (ref) & 2.912 & $2.244-3.769$ & \\
2 & & & 0.16 \\
3 & & & \\
$\geq 4$ & 1.445 & $1.211-1.786$ & 0.26 \\
Place of delivery & & & 0.36 \\
Private hospital (ref) & 0.775 & $0.411-1.376$ & 0.07 \\
Public hospital & 0.575 & $0.321-1.481$ & 0.003 \\
Source of information & 1.275 & $1.121-1.781$ & \\
Media (ref) & 2.122 & $1.956-2.897$ & \\
Neighbor & & & \\
CHW & & & \\
HCP & & & \\
& & & \\
\hline
\end{tabular}

Logistic regression was used to analyze the factors associated with knowledge of NDS (Table 5). The results show that those with secondary school or above were two times more knowledgeable than those who were not able to read and write $(\beta=2.245$, $\mathrm{CI}=1.924-2.882, \mathrm{p}=0.01)$. Participants with parity of 2-4 were nearly two times more knowledgeable $(\beta=1.978, \mathrm{CI}=1.675-2.322, \mathrm{p}=0.04)$, and those with five or more parity were more than two times more knowledgeable than those with parity of one $(\beta=2.118, \mathrm{CI}=1.975-2.72, \mathrm{p}=0.03)$. Participants with three ANC visits were nearly two times more knowledgeable $(\beta=1.987, C I=1.764-2.769, \mathrm{p}=0.02)$, and those with four ANC visits were three times more knowledgeable $(\beta=2.912, \quad C I=2.244-3.769$, $\mathrm{p}=0.01$ ) than those who attended only one ANC visit. Those who received information from HCP were two times more knowledgeable $(\beta=2.122, \quad \mathrm{CI}=1.956$ 2.897, $\mathrm{p}=0.00$ ) than those who received information from the media.

\section{DISCUSSION}

Parents knowledge of neonatal danger signs

The majority of participants were more knowledgeable of the NDS, not feeding well (44.0\%), similar to a study conducted in Baghdad.[10] Whereas the majority $(99 \%)$ were not knowledgeable of the NDS, >10 skin pustules or bullae, or swelling, redness, the hardness of skin, also similar to the study conducted in Baghdad.[10] In contrast, a study conducted in South West, Ethiopia by Asnakew,[11] showed that the most frequent NDS mentioned by participants, was fever $(82.8 \%)$, and less familiar NDS was lethargy (34.7\%).[11]

\section{Factors associated with parents' knowledge of neonatal danger signs}

This study showed that the HCP and CHW were significantly associated with parental knowledge of NDS. Similarly, a study conducted by Jemberia in Woldia General Hospital, Ethiopia, showed significant association with HCPs.[12] In contrast, studies conducted by Nigatu in North West of 
Ethiopia; and Asnakew in South West, Ethiopia, showed that HCPs were not significantly associated with maternal knowledge of NDS.[11,13]

The majority of participants that mentioned they were able to read and write $(64.7 \%)$ had no awareness of NDS, whereas $(24.5 \%)$ those with secondary school education were more knowledgeable of three NDS. Similarly, a community-based study conducted by Melkamu in Southwest Ethiopia with parents' knowledge of danger signs and health-seeking behavior showed that ANC attendance, paternal education, maternal education, paternal occupation, maternal occupation, and income were significantly associated with parents' knowledge.[14] Contrary to the study conducted by Ekwochi in Southeast Nigeria, maternal education was not significantly associated with knowledge of NDS.[15]

Concerning obstetrical factors and the number of ANC visits, those who attended two visits (62.6\%) had no awareness of NDS. In contrast, those who attended four visits $(35.8 \%)$ were more knowledgeable of four or more NDS. Those who delivered at home (33.3\%) had no awareness of NDS, while those who delivered at the health center $(30.1 \%)$ were more knowledgeable of two or more NDS. Similarly, Ayat found more than two-thirds had visited the clinic 3-6 times, which reflected good maternal awareness of NDS.[16] In contrast, Adem found that the number of ANC visits and parity were not considered significant factors with maternal awareness of NDS.[4]

The parity also was significantly associated with parents' knowledge of NDS. The results also showed that those with 2-4 parity were two times knowledgeable than those with one parity $(p=0.04)$, those with more than five parity were more than two times more knowledgeable than those with one parity $(p=0.03)$. Similarly, a study done by Ayat showed that parity, educational level, maternal age, and occupation were considered significant factors affected NDS. [16] Contrary to a study by Abdulrida, there was no significant relationship between the number of children and family support with seeking care outside the home, date and place of delivery with parents' knowledge of NDS.[10]

Parents' educational level was significantly associated with knowledge of NDS. The parents with secondary school and above were more than two times more knowledgeable than those who were not unable to read and write $(p=0.01)$. Similarly, a study done in Ethiopia showed that mothers with higher education were more knowledgeable compared to those with primary education.[16] In contrast, studies conducted by Ekwochi, [15] and Adem [4] showed no significant association with education. The possible justification for this finding could be that educated parents acquire knowledge through their academic life and experiences, and likely take their sick neonates to health facilities where they gain further information from HCP.

The number of ANC visits in this study was shown to be significantly associated with parents' knowledge of NDS. The participants with three ANC visits were two times more knowledgeable than those with one ANC visit $(p=0.02)$; those with 4 ANC visits were three times more knowledgeable than those with one ANC visit ( $p=0.01)$, similar to a study conducted by Nigatu.[13] In contrast, Adem showed that the number of ANC visits was not a significant factor.[4] The possible justification is that parents who attend ANC received more health education by HCPS and, therefore, able to retain and retrieve the knowledge at a later time.

The study showed that the place of delivery $(p=0.02)$ was significantly associated with parental knowledge of NDS. A similar study done by Molla showed that the place of delivery was significantly associated.[18] In contrast, the study by Adem showed that the place of delivery had no statistical association.[4] A likely explanation for this finding would be that increasing institutional birth improved the parents' knowledge of NDS due to exposure to health education by HCPs.

This study showed that parity was significantly associated with parents' knowledge of NDS. Those with 2-4 parity were two times knowledgeable than those with one parity $(\mathrm{p}=0.04)$, those with more than five parity were more than two times more knowledgeable than those with one parity $(p=0.03)$. This finding was similar to a study by Ayat.[16] In contrast, Abdulrida [10] and Adem [4] found no relationship between parity with mothers' knowledge about NDS. The likely justification is that as parity increased, so did the number of ANC visits and exposure to HCPs, postnatal services with $\mathrm{CHW}$, and valuable mothering experiences.

This study showed that HCPs as the source of information was significantly associated with parents' knowledge of NDS. Those who received 
information from HCP were two times more knowledgeable than those who received information from media $(p=0.003)$, similar to a study by Kibaru in Kenya.[19] In contrast, Nigatu revealed that exposure to the media, especially television, was increased mothers' knowledge of NDS.[13] This finding might be related to HCPs' commitment to teaching parents about neonatal health issues. Health education from HCPs provides that human touch to the message and likely increases the memorability compared to media.

\section{Limitations}

Data were collected from only four health centers located in the catchment area of a district hospital; therefore, the results cannot be generalized to all health centers in Rwanda. Another limitation is that only parents with neonates attending vaccination services were targeted for the study population, so the results cannot be generalized to all parents.

\section{Recommendations}

Further research is needed to assess the knowledge and associated factors of NDS among parents at the national level (all health centers). It is also recommended that faculty from the University of Rwanda Masters in Nursing faculty collaborate with the Ministry of Health and Ministry of Education to design specific educational and sensitization programs. Midwives and nurses in Rwanda could develop a Maternal Child Health Booklet, similar to the one in Kenya and about 30 other countries worldwide, to give to mothers at their first ANC visit, which could be shared with husbands for added knowledge retention. The valuable booklet provides vital health education about pregnancy, birth, and the infant, including information and pictures of signs and symptoms of NDS.[22]

\section{CONCLUSION}

The findings of this cross-sectional study indicated the need to enhance education of parents' knowledge of NDS in the study population. Parents' education level, parity, number of ANC visits, and HCPs as an informational source was significantly associated with parents' knowledge of NDS. The findings indicate the need to significantly increase educational efforts of NDS to parents both at the community and health facility level. Support and guidance at the national level are needed to initiate parental education to help meet the Sustainable Development Goals by 2030.

\section{Acknowledgments}

We acknowledge all participants that made this study successful.

This article is published open access under the Creative Commons Attribution-NonCommercial NoDerivatives (CC BYNC-ND4.0). People can copy and redistribute the article only for noncommercial purposes and as long as they give appropriate credit to the authors. They cannot distribute any modified material obtained by remixing, transforming or building upon this article. See https://creativecommons.org/licenses/by-ncnd $/ 4.0 /$

\section{REFERENCES}

1. World Health Organization. Newborns: reducing mortality. 2019(a). https://www.who.int/news-room/factsheets/detail/newborns-reducing-mortality.

2. UNICEF. Levels and trends in child mortality. Report 2019. Estimates developed by the UN Inter-agency Group for Child Mortality Estimation.Available from:https://www.unicef.org/media/60561/file/UN-IGME-child-mortalityreport-2019.pdf.

3. United States Agency for International Development. Recognition of Maternal and Newborns Complications. 2016; 67-75.

4. Rwanda Demographic and Health Survey (RDHS). 2014-2015; Available from: https://dhsprogram.com/pubs/pdf/FR316/FR316.pdf.

5. Uwingabire F, Gowan M. Birth asphyxia at a district hospital in Kigali, Rwanda. Rwanda Journal of Medicine and Health Sciences. 2019;2(2):104.

6. Gupta N, Hirschhorn LR, Rwabukwisi FC, Drobac P, Sayinzoga F, Mugeni C. Causes of death and predictors of childhood mortality in Rwanda: a matched case-control study using verbal social autopsy. BMC Public Health, 2018;18:1378. doi.org/10.1186/s12889-018-6282-z.

7. Price J, Lee J, Willcox M, Harnden A. Place of death, care-seeking and care pathway 
progression in the final illnesses of children under five years of age in sub-Saharan Africa: a systematic review. J Glob Health. 2019;9(2):020422.

8. World Health Organization. Integrated Management of Childhood Illness: management of the sick young infant aged up to 2 months. IMCI chart booklet. Geneva: World Health Organization; 2019(b). Licence: CC BY-NCSA 3.0 IGO. Available from: https://apps.who.int/iris/bitstream/handle/10665/326448/9789241516365eng.pdf?ua $=1$.

9. Adem N, Berhe KK, Tesfay. Awareness and associated factors towards neonatal danger signs among mothers attending public health institutions of Mekelle City, Tigray, Ethiopia, 2015. J Child Adolesc Behav, 2017. DOI:10.4172/2375-4494.1000365.

10. Kibaru EG, Otara AM. Knowledge of neonatal danger signs among mothers attending well baby clinic in Nakuru Central District, Kenya: cross-sectional descriptive study. BMC Res Notes. 2016; (December).

11. Sandberg J, Pettersson KO, Asp G, Kabakyenga J, Agardh A. Inadequate knowledge of neonatal danger signs among recently delivered women in Southwestern Rural Uganda: A community survey. 2014; 9 (5).

12. United Nations Development Programme (UNDP). From the MDGs to Sustainable Development for all. Lessons from 15 years of practice. 2016; 67-79. Available from:https://www.undp.org/content/dam/undp/library/SDGs/English/From\%20the\%20MDGs\%20to\%20SD4 All.pdf.

13. Abdulrida HN, Hassan RJ, Sabri MM. Knowledge and health-seeking practices of mothers attending primary health-care centers in Baghdad Al-Karkh sector about danger signs in newborns. Mustansiriya Medical Journal, 2018;17(1):29-35.

14. Asnakew DT, Engidaw MT, Gebremariam AD. Level of knowledge about neonatal danger signs and associated factors among mothers who delivered at home in Fogera District, South West, Ethiopia. 2019; 3(4):53-60.

15. Jemberia MM, Berhe ET, Mirkena HB, Gishen DM, Tegegne AE, Reta MA. Low level of knowledge about neonatal danger signs and its associated factors among postnatal mothers attending at Woldia general hospital, Ethiopia. 2018; 1-8.

16. Nigatu SG, Worku AG, Dadi AF. Level of mother's knowledge about neonatal danger signs and associated factors in North West of Ethiopia: a community based study. BMC Res Notes. 2015; 4-9.

17. Melkamu B, Berhane M, Yimam H, Jibat N, Zewdu M. Parents ' Knowledge of Danger Signs and Health Seeking Behavior in Newborn and Young Infant Illness in Tiro Afeta District, Southwest Ethiopia: A Communitybased Study. 2018 (1):47-49.

18. Ekwochi U, Ndu IK, Osuorah CDI, Amadi OF, Okeke IB, Obuoha E, et al. Knowledge of danger signs in newborns and health-seeking practices of mothers and caregivers in Enugu state, Southeast Nigeria. Ital J Pediatr. 2015;41:18. doi:10.1186/s13052-0150127-5.

19. Massoud A, Hussein AA, Ahmad ER. Factors associated with neonatal danger signs among high-risk mothers during the perinatal period. 2019;9(4):17-28.

20. Anmut W, Fekecha B, Demeke T. Mother's knowledge and Practice about Neonatal Danger Signs. 2017; 1-7.

21. Molla G, Gonie A, Belachew T, Admasu B. Health care-seeking behaviour on neonatal danger signs among mothers in Tenta District, Northeast Ethiopia: Community based cross-sectional study. 2017; 9 (July):85-93.

22. Mudany MA, Sirengo M, Rutherford GW, Mwangi M, Nganga LW, Gichangi A. Enhancing Maternal and Child Health using a combined Mother \& Child Health Booklet in Kenya. 2015;(September):442-7. 